\title{
Quantum billiards with branes on product of Einstein spaces
}

\author{
V. D. Ivashchuk ${ }^{1,2, a}$ \\ ${ }^{1}$ Center for Gravitation and Fundamental Metrology, VNIIMS, Ozyornaya St., 46, Moscow 119361, Russia \\ ${ }^{2}$ Institute of Gravitation and Cosmology, Peoples' Friendship University of Russia, Miklukho-Maklaya St.,6, Moscow 117198, Russia
}

Received: 25 April 2016 / Accepted: 10 May 2016 / Published online: 21 May 2016

(C) The Author(s) 2016. This article is published with open access at Springerlink.com

\begin{abstract}
We consider a gravitational model in dimension $D$ with several forms, $l$ scalar fields and a $\Lambda$-term. We study cosmological-type block-diagonal metrics defined on a product of an 1-dimensional interval and $n$ oriented Einstein spaces. As an electromagnetic composite brane ansatz is adopted and certain restrictions on the branes are imposed the conformally covariant Wheeler-DeWitt (WDW) equation for the model is studied. Under certain restrictions, asymptotic solutions to the WDW equation are found in the limit of the formation of the billiard walls. These solutions reduce the problem to the so-called quantum billiard in $(n+l-1)$ dimensional hyperbolic space. Several examples of quantum billiards in the model with electric and magnetic branes, e.g. corresponding to hyperbolic Kac-Moody algebras, are considered. In the case $n=2$ we find a set of basis asymptotic solutions to the WDW equation and derive asymptotic solutions for the metric in the classical case.
\end{abstract}

\section{Introduction}

In this paper we deal with the quantum billiard approach to $D$-dimensional cosmological-type models defined on a (warped) product manifold $\left(u_{-}, u_{+}\right) \times M_{1} \times \cdots \times M_{n}$, where $M_{i}$ is a smooth oriented Einstein manifold, $i=1, \ldots, n$.

The billiard approach in classical gravity originally appeared in the dissertation of Chitré [1] for an explanation of the BKL oscillations $[2,3]$ while approaching to a spacelike singularity in the Bianchi-IX model [4]. In this description a simple triangle billiard in the hyperbolic (Lobachevsky) space $H^{2}$ was used. The BKL-like behavior near a timelike singularity was studied in $[5,6]$.

In [7], the billiard approach for $D=4$ was extended to the quantum case (see also [8]), i.e. the solutions to the WheelerDeWitt (WDW) equation [9] were reduced to the problem of

\footnotetext{
a e-mail: ivashchuk@mail.ru
}

finding the spectrum of the Laplace-Beltrami operator on Chitré's triangle billiard.

Chitré's billiard approach was generalized to a multidimensional cosmological model with multicomponent anisotropic ("perfect") fluid [10-12] defined on the product of $n$ Einstein factor spaces and 1-dimensional manifold. The search for an oscillating behavior near the singularity was reduced to the problem of proving the finiteness of the billiard volume. At that time the quantum billiard approach to multidimensional cosmology was suggested in [12-14].

The classical billiard approach to multidimensional models with fields of forms and scalar fields in the presence of the $\Lambda$-term was suggested in [15] along lines suggested earlier in [12]. In Ref. [15] rather a general composite electromagnetic ansatz for the fields of forms on a warped product of several Einstein manifolds and a 1-dimensional base manifold $M_{0}$ was developed. Reference [15] contained rather a general construction of the billiard approach for the description of the behavior of scale factors and scalar fields near either a spacelike or a timelike singularity, i.e. the metric in [15] $\mathrm{d} s^{2}=w \mathrm{~d} u^{2}+\cdots$, contained an arbitrary sign $w= \pm 1$ and a coordinate $u$. Thus, the paper [15] was dealing with cosmological-type solutions, e.g. cosmological, spherically symmetric, and cylindrically symmetric ones. The metric had a block-diagonal form. In [15] the necessary condition for the formation of walls was formulated in terms of inequalities for scalar products of the brane vectors $U^{s}:\left(U^{s}, U^{s}\right)>0$ and for the so-called brane sign parameters: $\varepsilon_{s}>0$. Inequalities on Kasner parameters, where formulated in terms of linear functions $U^{s}(\alpha)$, which give either Kasner or oscillatory asymptotic regimes near the singularity. Another advantage of the approach of [15] was in dealing with a wide variety of signatures of Einstein factor space metrics (though restricted by $\varepsilon_{s}>0$ ). It was shown that the curvatures of the Einstein factor spaces and the $\Lambda$-term are irrelevant near the singularity.

Meanwhile the approach of Ref. [15] had some restrictive points, since it was dealing with block-diagonal metrics and 
putting restrictions on brane intersections (for branes corresponding to the same form field) which guaranteed blockdiagonal structure of the stress-energy tensor. For some extension of these restrictions see Ref. [16].

Some problems of the approach of Ref. [15] were overcome in the papers of Damour et al. [17-22] and some other authors. These works were aimed from the very beginning at studying the generic behavior of solutions near a spacelike singularity (a lá BKL) for gravitational and cosmological models with non-diagonal metrics, fields of forms and scalar fields. This approach was based on a wide use of Iwasawa decomposition and hyperbolic Kac-Moody algebras [23-26]. It was shown in [21] that for certain models (of supergravity) the billiards (or their parts) are related to Weyl chambers of certain hyperbolic Kac-Moody (KM) algebras. This fact has simplified the proof of the finiteness of the billiard volume in certain cases.

In the recent publications [27-29] the quantum billiard approach for the multidimensional gravitational model with several forms was considered. The main motivation for the quantum billiard approach in $[27,28]$ was coming from the quantum gravity paradigm; see [30] and references therein. The asymptotic solutions to the WDW equation from $[27,28]$ (in the model without scalar fields) are similar to those obtained earlier in [12] for a multicomponent anisotropic fluid with certain equations of state. In [29] another, conformally covariant form of the WDW equation [31-35] was used. In this case the minisuperspace was enlarged by including the form potentials for electric non-composite branes. In [29] an example of a 9-dimensional quantum billiard for $D=11$ model with 120 four-forms which mimic spacelike $M 2$-brane solutions (SM2-branes in $D=11$ supergravity) was considered. It was shown that the wave function vanishes as $y^{0} \rightarrow-\infty$ (i.e. at the singularity), where $y^{0}$ is the "tortoise" timelike coordinate in minisuperspace [29]. In [36] we have generalized the approach of [29] to the case when scalar fields with dilatonic couplings were added into consideration and the composite electromagnetic ansatz for branes was considered instead of the non-composite electric one from [29]. New examples of quantum billiards with electric and magnetic $S$-branes in $D=11$ and $D=10$ models were presented. It was found that in the quantum case adding of magnetic branes changes the asymptotic behavior of the wave functions, while it does not change the classical asymptotic oscillating behavior of the scale factors (and scalar field for $D=10$ ). It was found that in certain examples the basis wave functions in the "tortoise gauge" vanish as $y^{0} \rightarrow-\infty$.

In this paper we generalize the approach from [36] to $n$ Einstein factor spaces and a $\Lambda$ term. We also extend this approach by relaxing the main restriction for brane vectors: $\left(U^{s}, U^{s}\right)>0$. Here we consider examples of billiards in the model with $n$ non-intersecting electric branes, $n \geq 2$. The brane world volumes are volume forms of $M_{i}$. We show that in the classical case any of these billiards describe the never ending oscillating behavior of the scale factors while approaching a singularity, which may be either spacelike or timelike one. The examples with timelike singularities are supported by the use of either phantom form fields, or extra time-variables. For $n=2$ (when 1-dimensional $M_{1}$ and $M_{2}$ are forbidden) we obtain the basis asymptotic solutions to the WDW equation.

We also generalize the model with $n$ electric branes by adding a form of rank $D$. This adding does not change the billiard but it changes (e.g. drastically for $D \leq 7$ ) the basis asymptotic solutions to the WDW equation for a certain choice of Bessel function.

Here we also consider an example of a 4-dimensional quantum billiard in $D=11$ model with ten 4 -forms [15]. We use the ansatz with ten magnetic non-composite branes with brane world volumes of the form $M_{i} \times M_{j} \times M_{k}(i<j<k)$, where $M_{i}$ is $2 d$ Einstein space, $i=1, \ldots, 5$. We prove the vanishing of the basis wave functions in the "tortoise gauge" as $y^{0} \rightarrow-\infty$.

\section{The model}

Here we study the multidimensional gravitational model governed by the action

$S_{\text {act }}=\frac{1}{2 \kappa^{2}} \int_{M} \mathrm{~d}^{D} z \sqrt{|g|} \mathcal{L}+S_{\mathrm{YGH}}$,

where

$$
\begin{aligned}
\mathcal{L}= & R[g]-2 \Lambda+h_{\alpha \beta} g^{M N} \partial_{M} \varphi^{\alpha} \partial_{N} \varphi^{\beta} \\
& -\sum_{a \in \Delta} \frac{\theta_{a}}{n_{a} !} \exp \left[2 \lambda_{a}(\varphi)\right]\left(F^{a}\right)_{g}^{2},
\end{aligned}
$$

$g=g_{M N}(z) \mathrm{d} z^{M} \otimes \mathrm{d} z^{N}$ is a metric on the manifold $M$, $\operatorname{dim} M=D, \Lambda$ is cosmological constant, $\varphi=\left(\varphi^{\alpha}\right) \in \mathbb{R}^{l}$ is a vector of scalar fields, $\left(h_{\alpha \beta}\right)$ is a non-degenerate symmetric $l \times l$ matrix $(l \in \mathbb{N}), \theta_{a} \neq 0$, and

$F^{a}=d A^{a}=\frac{1}{n_{a} !} F_{M_{1} \ldots M_{n_{a}}}^{a} d z^{M_{1}} \wedge \cdots \wedge d z^{M_{n_{a}}}$

is an $n_{a}$-form $\left(n_{a} \geq 2\right)$ on $M$ and $\lambda_{a}$ is a 1 -form on $\mathbb{R}^{l}$ : $\lambda_{a}(\varphi)=\lambda_{a \alpha} \varphi^{\alpha}, a \in \Delta, \alpha=1, \ldots, l$. In (2.1) we denote $|g|=\left|\operatorname{det}\left(g_{M N}\right)\right|,\left(F^{a}\right)_{g}^{2}=F_{M_{1} \ldots M_{n_{a}}}^{a} F_{N_{1} \ldots N_{n_{a}}}^{a} g^{M_{1} N_{1}} \ldots$ $g^{M_{n_{a}} N_{n_{a}}}, a \in \Delta$, where $\Delta$ is some finite set of (color) indices and $S_{\mathrm{YGH}}$ is the standard (York-Gibbons-Hawking) boundary term. In the models with one time and the usual fields of forms all $\theta_{a}$ obey $\theta_{a}>0$ when the signature of the metric is $(-1,+1, \ldots,+1)$. For such a choice of signature $\theta_{b}<0$ corresponds to a "phantom" form field $F^{b}$. 
We consider the manifold

$M=\mathbb{R}_{*} \times M_{1} \times \cdots \times M_{n}$,

with the metric

$g=w e^{2 \gamma(u)} \mathrm{d} u \otimes \mathrm{d} u+\sum_{i=1}^{n} e^{2 \beta^{i}(u)} g^{i}$,

where $\mathbb{R}_{*}=\left(u_{-}, u_{+}\right), w= \pm 1$, and $g^{i}=g_{m_{i} n_{i}}^{i}\left(y_{i}\right) \mathrm{d} y_{i}^{m_{i}} \otimes$ $\mathrm{d} y_{i}^{n_{i}}$ is an Einstein metric on $M_{i}$ satisfying the equation

$R_{m_{i} n_{i}}\left[g^{i}\right]=\xi_{i} g_{m_{i} n_{i}}^{i}$,

$m_{i}, n_{i}=1, \ldots, d_{i} ; \xi_{i}$ is constant, $i=1, \ldots, n$. The functions $\gamma, \beta^{i}: \mathbb{R}_{*} \rightarrow \mathbb{R}$ are smooth. We denote $d_{i}=\operatorname{dim} M_{i}$; $i=1, \ldots, n$ and $d_{0}=1 ; D=\sum_{\nu=0}^{n} d_{\nu}$. We put any manifold $M_{i}, i=1, \ldots, n$, taking it to be oriented and connected. Then the volume $d_{i}$-form

$\tau_{i} \equiv \sqrt{\left|g^{i}\left(y_{i}\right)\right|} \mathrm{d} y_{i}^{1} \wedge \cdots \wedge \mathrm{d} y_{i}^{d_{i}}$,

and signature parameter

$\varepsilon(i) \equiv \operatorname{sign}\left(\operatorname{det}\left(g_{m_{i} n_{i}}^{i}\right)\right)= \pm 1$

are correctly defined for all $i=1, \ldots, n$.

The cosmological ( $S$-brane) solutions correspond to $w=$ -1 and positive definite $g^{i}$ for all $i$, while static configurations (e.g. fluxbranes, wormholes, black branes etc.) may be obtained when $w=1, g^{k}$ are Riemannian metrics for all $k>1$, and $g^{1}$ is the metric of pseudo-Euclidean signature $(-,+, \ldots,+)$. Here we may also deal with solutions having several timelike directions.

By $\Omega=\Omega(n)$ we denote the set of all non-empty subsets of $\{1, \ldots, n\}$. For any $I=\left\{i_{1}, \ldots, i_{k}\right\} \in \Omega, i_{1}<\cdots<i_{k}$, we denote

$\tau(I) \equiv \tau_{i_{1}} \wedge \cdots \wedge \tau_{i_{k}}$

$\varepsilon(I) \equiv \varepsilon\left(i_{1}\right) \ldots \varepsilon\left(i_{k}\right)$,

$d(I) \equiv \sum_{i \in I} d_{i}$.

For fields of forms we consider the following composite electromagnetic ansatz:

$F^{a}=\sum_{I \in \Omega_{a, e}} \mathcal{F}^{(a, e, I)}+\sum_{J \in \Omega_{a, m}} \mathcal{F}^{(a, m, J)}$,

where

$\mathcal{F}^{(a, e, I)}=d \Phi^{(a, e, I)} \wedge \tau(I)$,

$\mathcal{F}^{(a, m, J)}=e^{-2 \lambda_{a}(\varphi)} *\left(d \Phi^{(a, m, J)} \wedge \tau(J)\right)$ are elementary forms of electric and magnetic types, respectively, $a \in \Delta, I \in \Omega_{a, e}, J \in \Omega_{a, m}$, and $\Omega_{a, v} \subset \Omega, v=e, m$. In $(2.13) *=*[g]$ is the Hodge operator on $(M, g)$.

For scalar functions we put

$\varphi^{\alpha}=\varphi^{\alpha}(u), \quad \Phi^{s}=\Phi^{s}(u)$,

$s \in S$. Thus, $\varphi^{\alpha}$ and $\Phi^{s}$ are functions on $\left(u_{-}, u_{+}\right)$.

Here and below the set $S$ consists of elements

$s=\left(a_{s}, v_{s}, I_{s}\right)$,

where $a_{s} \in \Delta$ is the color index, $v_{s}=e, m$ is the electromagnetic index, and the set $I_{s} \in \Omega_{a_{s}, v_{s}}$ describes the location of the brane.

Due to (2.12) and (2.13) we get $d(I)=n_{a}-1, \quad d(J)=$ $D-n_{a}-1$.

Here we present two restrictions on the sets of branes which guarantee the diagonal form of the energy-momentum tensor [37].

The first restriction for a pair of two (different) branes both electric (ee-pair) or magnetic ( $\mathrm{mm}$-pair) with coinciding color index reads

$\mathrm{d}(I \cap J) \leq \mathrm{d}(I)-2$,

for any $I, J \in \Omega_{a, v}, a \in \Delta, v=e, m$ (here $\mathrm{d}(I)=\mathrm{d}(J)$ ).

The second restriction for any pair of two branes with the same color index, which include one electric and one magnetic brane (em-pair) has the following form:

$\mathrm{d}(I \cap J) \neq 0$,

where $I \in \Omega_{a, e}, J \in \Omega_{a, m}, a \in \Delta$.

These restrictions are satisfied identically in the noncomposite case, when there are no two branes corresponding to the same form $F^{a}$ for any $a \in \Delta$.

It follows from [37] that the equations of motion for the model (2.1) and the Bianchi identities, $\mathrm{d} \mathcal{F}^{s}=0, s \in S_{m}$, for fields from (2.4), (2.11)-(2.14), when restrictions $(R 1)$ and $(R 2)$ are imposed, are equivalent to the equations of motion for the $\sigma$-model governed by the action

$S_{\sigma}=\frac{\mu}{2} \int \mathrm{d} u \mathcal{N}\left\{\mathcal{G}_{\hat{A} \hat{B}}(X) \dot{X}^{\hat{A}} \dot{X}^{\hat{B}}-2 \mathcal{N}^{-2} V_{w}\right\}$,

where $X=\left(X^{\hat{A}}\right)=\left(\beta^{i}, \varphi^{\alpha}, \Phi^{S}\right) \in \mathbb{R}^{N}, N=n+l+m$, $m=|S|$ is the number of branes, and the minisupermetric $\mathcal{G}=\mathcal{G}_{\hat{A} \hat{B}}(X) \mathrm{d} X^{\hat{A}} \otimes \mathrm{d} X^{\hat{B}}$ on the minisuperspace $\mathcal{M}=\mathbb{R}^{N}$ is defined as follows:

$\left(\mathcal{G}_{\hat{A} \hat{B}}(X)\right)=\left(G_{i j}, h_{\alpha \beta}, \varepsilon_{s} \exp \left(-2 U^{s}(\sigma)\right) \delta_{s s^{\prime}}\right)$. 
where $\dot{x} \equiv \mathrm{d} x / \mathrm{d} u,\left(\sigma^{A}\right)=\left(\beta^{i}, \varphi^{\alpha}\right), k_{0} \neq 0$, the index set $S$ is defined in (2.15),

$$
\left(\hat{G}_{A B}\right)=\operatorname{diag}\left(G_{i j}, h_{\alpha \beta}\right)
$$

is the truncated target space metric with

$$
G_{i j}=d_{i} \delta_{i j}-d_{i} d_{j},
$$

and the co-vectors

$$
\begin{aligned}
& U^{s}=U_{A}^{s} \sigma^{A}=\sum_{i \in I_{s}} d_{i} \beta^{i}-\chi_{s} \lambda_{a_{s}}(\varphi), \\
& \left(U_{A}^{s}\right)=\left(d_{i} \delta_{i} I_{s},-\chi_{s} \lambda_{a_{s} \alpha}\right), \\
& s=\left(a_{s}, v_{s}, I_{s}\right), \\
& V_{w}=-w \Lambda e^{2 \gamma_{0}(\beta)}+\frac{w}{2} \sum_{i=1}^{n} \xi_{i} d_{i} e^{-2 \beta^{i}+2 \gamma_{0}(\beta)}
\end{aligned}
$$

is the potential with $\gamma_{0}(\beta) \equiv \sum_{i=1}^{n} d_{i} \beta^{i}$, and $\mathcal{N}=\exp \left(\gamma_{0}-\right.$ $\gamma)>0$ is the modified lapse function.

We denote $\chi_{e}=+1$ and $\chi_{m}=-1$;

$$
\delta_{i I}=\sum_{j \in I} \delta_{i j}
$$

is the indicator of $i$ belonging to $I: \delta_{i I}=1$ for $i \in I$ and $\delta_{i I}=0$ otherwise; and

$\varepsilon_{s}=\varepsilon\left(I_{s}\right) \theta_{a_{s}}$ for $v_{s}=e ; \quad \varepsilon_{s}=-\varepsilon[g] \varepsilon\left(I_{s}\right) \theta_{a_{s}}$ for $v_{s}=m$,

$s \in S, \varepsilon[g] \equiv \operatorname{sign} \operatorname{det}\left(g_{M N}\right)$

In the electric case $\left(\mathcal{F}^{(a, m, I)}=0\right)$ for finite internal space volumes $V_{i}$ the action (2.18) coincides with the action (2.1) if $\mu=-w / \kappa_{0}^{2}, \kappa^{2}=\kappa_{0}^{2} V_{1} \ldots V_{n}$.

In the following we will use the scalar products of $U^{s}$. vectors $\left(U^{s}, U^{s^{\prime}}\right) ; s, s^{\prime} \in S$, where

$\left(U, U^{\prime}\right)=\hat{G}^{A B} U_{A} U_{B}^{\prime}$,

for $U=\left(U_{A}\right), U^{\prime}=\left(U_{A}^{\prime}\right) \in \mathbb{R}^{N_{0}}, N_{0}=n+l$, and

$\left(\hat{G}^{A B}\right)=\operatorname{diag}\left(G^{i j}, h^{\alpha \beta}\right)$

is the matrix inverse to the matrix (2.20). Here (as in [33])

$G^{i j}=\frac{\delta^{i j}}{d_{i}}+\frac{1}{2-D}$,

$i, j=1, \ldots, n$.
The scalar products (2.26) read [37]

$\left(U^{s}, U^{s^{\prime}}\right)=\mathrm{d}\left(I_{s} \cap I_{s^{\prime}}\right)+\frac{\mathrm{d}\left(I_{S}\right) \mathrm{d}\left(I_{s^{\prime}}\right)}{2-D}+\chi_{s} \chi_{s^{\prime}} \lambda_{a_{s} \alpha} \lambda_{a_{s^{\prime}} \beta} h^{\alpha \beta}$,

where $\left(h^{\alpha \beta}\right)=\left(h_{\alpha \beta}\right)^{-1}$ and $s=\left(a_{s}, v_{s}, I_{s}\right), s^{\prime}=$ $\left(a_{s^{\prime}}, v_{s^{\prime}}, I_{s^{\prime}}\right)$ belong to $S$.

The potential (2.23) reads as follows:

$V_{w}=(-w \Lambda) \mathrm{e}^{2 U^{\Lambda}(\sigma)}+\sum_{j=1}^{n} \frac{w}{2} \xi_{j} d_{j} \mathrm{e}^{2 U^{(j)}(\sigma)}$,

where

$U^{(j)}(\sigma)=U_{A}^{(j)} \sigma^{A}=-\beta^{j}+\gamma_{0}(\beta)$,

$\left(U_{A}^{(j)}\right)=\left(-\delta_{i}^{j}+d_{i}, 0\right)$,

$U^{\Lambda}(\sigma)=U_{A}^{\Lambda} \sigma^{A}=\gamma_{0}(\beta), \quad\left(U_{A}^{\Lambda}\right)=\left(d_{i}, 0\right)$.

The scalar products of co-vectors $U^{\Lambda}, U^{(j)}, U^{s}$ are defined by the following relations [37]:

$\left(U^{(i)}, U^{(j)}\right)=\frac{\delta_{i j}}{d_{j}}-1$,

$\left(U^{(i)}, U^{\Lambda}\right)=-1, \quad\left(U^{(i)}, U^{s}\right)=-\delta_{i I_{s}}$,

$\left(U^{s}, U^{\Lambda}\right)=\frac{\mathrm{d}\left(I_{s}\right)}{2-D}, \quad\left(U^{\Lambda}, U^{\Lambda}\right)=-\frac{D-1}{D-2}$,

where $s=\left(a_{s}, v_{s}, I_{S}\right) \in S ; i, j=1, \ldots, n$.

The vector $U^{\Lambda}$ is a timelike as well as $U^{(i)}$ with $d_{i}>1$ (here we deal with $U^{(i)}$ obeying $\xi_{i} \neq 0$ ). The vectors $U^{\Lambda}$ and $U^{(i)}$ with $d_{i}>1$ belong to the same light cone (interior part) due to the relations $\left(U^{(i)}, U^{\Lambda}\right)=-1$.

\section{Quantum billiard approach}

Here we generalize the quantum billiard approach for asymptotic solutions to the Wheeler-DeWitt (WDW) equation from [36] to the case of a chain of Einstein spaces in the presence of the $\Lambda$-term.

Let us denote by $S_{+}$the subset of all $s \in S$ obeying

$\left(U^{s}, U^{s}\right)=\mathrm{d}\left(I_{S}\right)\left(1+\frac{\mathrm{d}\left(I_{S}\right)}{2-D}\right)+\lambda_{a_{s} \alpha} \lambda_{a_{s} \beta} h^{\alpha \beta}>0$.

First we put the following additional restrictions on the model:

(i) $\left(h_{\alpha \beta}\right)>0$,

(ii) $\varepsilon_{s}>0$ for all $s \in S_{+}$.

These restrictions are necessary conditions for the formation of infinite "wall" potential in hyperbolic spaces in certain limit (see below). The first restriction excludes phantom 
scalar fields. We note that in our previous work [29,36,38] we used a more rigid restriction: $S=S_{+}$.

By fixing the temporal gauge:

$\gamma_{0}-\gamma=2 f(X), \quad \mathcal{N}=e^{2 f}$,

where $f: \mathcal{M} \rightarrow \mathbb{R}$ is a smooth function, we obtain the Lagrange system with the Lagrangian

$L_{f}=\frac{\mu}{2} \mathrm{e}^{2 f} \mathcal{G}_{\hat{A} \hat{B}}(X) \dot{X}^{\hat{A}} \dot{X}^{\hat{B}}-\mu \mathrm{e}^{-2 f} V_{w}$

and the energy constraint

$E_{f}=\frac{\mu}{2} \mathrm{e}^{2 f} \mathcal{G}_{\hat{A} \hat{B}}(X) \dot{X}^{\hat{A}} \dot{X}^{\hat{B}}+\mu \mathrm{e}^{-2 f} V_{w}=0$.

The set of Lagrange equations with the constraint (3.6) is equivalent to the set of Hamiltonian equations for the Hamiltonian

$H^{f}=\frac{1}{2 \mu} \mathrm{e}^{-2 f} \mathcal{G}^{\hat{A} \hat{B}}(X) P_{\hat{A}} P_{\hat{B}}+\mu \mathrm{e}^{-2 f} V_{w}$

with the constraint

$H^{f}=0$,

where $P_{\hat{A}}=\mu \mathrm{e}^{2 f} \mathcal{G}_{\hat{A} \hat{B}}(X) \dot{X}^{\hat{B}}$ are momenta (for fixed gauge) and $\left(\mathcal{G}^{\hat{A} \hat{B}}\right)=\left(\mathcal{G}_{\hat{A} \hat{B}}\right)^{-1}$.

Here we use the prescriptions of covariant and conformally covariant quantization of the hamiltonian constraint $H^{f}=0$, which was suggested initially by Misner [31] and considered afterwards in $[32,33,35]$ and some other papers.

We obtain the Wheeler-DeWitt (WDW) equation,

$\hat{H}^{f} \Psi^{f} \equiv\left(-\frac{1}{2 \mu} \Delta\left[e^{2 f} \mathcal{G}\right]+\frac{a}{\mu} R\left[e^{2 f} \mathcal{G}\right]+\mu \mathrm{e}^{-2 f} V_{w}\right)$

$\Psi^{f}=0$,

where

$a=a_{N}=\frac{(N-2)}{8(N-1)}$,

$N=n+l+m$.

Here $\Psi^{f}=\Psi^{f}(X)$ is the wave function corresponding to the $f$-gauge (3.4) and satisfying the relation

$\Psi^{f}=e^{b f} \Psi^{f=0}, \quad b=b_{N}=(2-N) / 2$.

In (3.9) we denote by $\Delta\left[\mathcal{G}^{f}\right]$ and $R\left[\mathcal{G}^{f}\right]$ the LaplaceBeltrami operator and the scalar curvature corresponding to the metric

$\mathcal{G}^{f}=e^{2 f} \mathcal{G}$,

respectively.
The Wheeler-DeWitt (WDW) equation (3.9) is conformally covariant. This follows from (3.11) and the relation

$\hat{H}^{f}=e^{-2 f} e^{b f} \hat{H}^{f=0} e^{-b f}$,

where the coefficients $a_{N}$ and $b_{N}$ are well known in the conformally covariant theory of scalar field.

Now we put $f=f(\sigma)$ and denote

$\bar{U}=\sum_{s \in S} \bar{U}^{s}, \quad \bar{U}^{s}=U^{s}(\sigma)-f$

and

$\bar{G}_{A B}=e^{2 f} \hat{G}_{A B}, \quad \bar{G}^{A B}=e^{-2 f} \hat{G}^{A B}$.

Here we deal with a special class of asymptotic solutions to the WDW equation. Due to restrictions (3.2) and (3.3) the (minisuperspace) metrics $\hat{G}, \mathcal{G}$ have pseudo-Euclidean signatures $(-,+, \ldots,+)$. We put $f=f_{0}$, where

$e^{2 f_{0}}=-\left(\hat{G}_{A B} \sigma^{A} \sigma^{B}\right)^{-1}$,

and we impose $\hat{G}_{A B} \sigma^{A} \sigma^{B}<0$. With this choice we deal with the so-called "tortoise" time gauge.

Here we use a diagonalization of $\sigma$-variables

$\sigma^{A}=S_{a}^{A} z^{a}$

$a=0, \ldots, N_{0}-1$, with $N_{0}=n+l$, obeying $\hat{G}_{A B} \sigma^{A} \sigma^{B}=$ $\eta_{a b} z^{a} z^{b}$, where $\left(\eta_{a b}\right)=\operatorname{diag}(-1,+1, \ldots,+1)$.

We restrict the WDW equation to the lower light cone $V_{-}=\left\{z=\left(z^{0}, \vec{z}\right) \mid z^{0}<0, \eta_{a b} z^{a} z^{b}<0\right\}$, and we introduce the Misner-Chitré-like coordinates

$\begin{aligned} z^{0} & =-e^{-y^{0}} \frac{1+\vec{y}^{2}}{1-\vec{y}^{2}}, \\ \vec{z} & =-2 e^{-y^{0}} \frac{\vec{y}}{1-\vec{y}^{2}},\end{aligned}$

where $y^{0}<0$ and $\vec{y}^{2}<1$.

In these variables we have $f_{0}=y^{0}$. In the following we use

$\bar{G}=-\mathrm{d} y^{0} \otimes \mathrm{d} y^{0}+h_{L}$,

where

$h_{L}=\frac{4 \delta_{r s} \mathrm{~d} y^{r} \otimes \mathrm{d} y^{s}}{\left(1-\vec{y}^{2}\right)^{2}}$

(the summation over $r, s=1, \ldots, N_{0}-1$ is assumed). The metric $h_{L}$ is defined on the unit ball $D^{N_{0}-1}=\{\vec{y} \in$ $\left.\mathbb{R}^{N_{0}-1} \mid \vec{y}^{2}<1\right\} . D^{N_{0}-1}$ with the metric $h_{L}$ is a realization of the hyperbolic space $H^{N_{0}-1}$. 
For the wave function we consider the ansatz from [36]:

$\Psi^{f_{0}}=e^{C(\sigma)} e^{i Q_{s} \Phi^{s}} \Psi_{0, L}(\sigma)$,

where

$C(\sigma)=\frac{1}{2} \bar{U}=\frac{1}{2}\left(\sum_{s \in S} U_{A}^{s} \sigma^{A}-m f_{0}\right)$,

where $Q_{s} \neq 0$ and $e^{i Q_{s} \Phi^{s}}=\exp \left(i \sum_{s \in S} Q_{s} \Phi^{s}\right)$.

Repeating all calculations from [36] we get

$$
\begin{aligned}
& \hat{H}^{f_{0}} \Psi^{f_{0}}=\mu^{-1} e^{C(\sigma)} e^{i Q_{s} \Phi^{s}}\left(-\frac{1}{2} \Delta[\bar{G}]\right. \\
& \left.\quad+\frac{1}{2} \sum_{s \in S} Q_{s}^{2} e^{-2 f_{0}+2 U^{s}(\sigma)}+\delta V+\mu^{2} e^{-2 f_{0}} V_{w}\right) \Psi_{0, L}=0,
\end{aligned}
$$

where

$\delta V=A e^{-2 f_{0}}-\frac{1}{8}(n+l-2)^{2}$

and

$A=\frac{1}{8(N-1)}\left[\sum_{s, s^{\prime} \in S}\left(U^{s}, U^{s^{\prime}}\right)-(N-2) \sum_{s \in S}\left(U^{s}, U^{s}\right)\right]$.

Here and in the following $U^{s}(\sigma)=U_{A}^{s} \sigma^{A}$.

Now we proceed with the study the asymptotic solutions to WDW equation in the limit $y^{0} \rightarrow-\infty$. Due to (3.22) and (3.24) this equation reads

$\left(-\frac{1}{2} \Delta[\bar{G}]+\frac{1}{2} \sum_{s \in S} Q_{s}^{2} e^{-2 f_{0}+2 U^{s}(\sigma)}+\delta V+\mu^{2} e^{-2 f_{0}} V_{w}\right)$

$\Psi_{0, L}=0$.

Here and in the following we put $Q_{s} \neq 0$ for all $s \in S_{+}$.

It follows from the analysis of [15] that for a certain choice of diagonalization (3.17),

$\frac{1}{2} \sum_{s \in S} Q_{s}^{2} e^{-2 f_{0}+2 U^{s}(\sigma)} \rightarrow V_{\infty}$

$e^{-2 f_{0}} \mu^{2} V_{w} \rightarrow 0$

as $y^{0}=f_{0} \rightarrow-\infty$. Here $V_{\infty}$ is the potential of infinite walls which are produced by branes with $\left(U^{s}, U^{s}\right)>0$ :

$V_{\infty}=\sum_{s \in S_{+}} \theta_{\infty}\left(\vec{v}_{s}^{2}-1-\left(\vec{y}-\vec{v}_{s}\right)^{2}\right)$, where we denote $\theta_{\infty}(x)=+\infty$, for $x \geq 0$ and $\theta_{\infty}(x)=0$ for $x<0$. The vectors $\vec{v}_{s}, s \in S_{+}$, which belong to $\mathbb{R}^{N_{0}-1}$ $\left(N_{0}=n+l\right)$, are defined by

$\vec{v}_{s}=-\vec{u}^{s} / u_{0}^{s}$

where the $N_{0}$-dimensional vectors $u^{s}=\left(u_{0}^{s}, \vec{u}^{s}\right)=\left(u_{a}^{s}\right)$ are obtained from brane $U^{s}$-vectors using the matrix $\left(S_{a}^{A}\right)$ from (3.17)

$u_{a}^{s}=S_{a}^{A} U_{A}^{s}$.

By definition of $S_{+}$we get

$\left(U^{s}, U^{s}\right)=-\left(u_{s 0}\right)^{2}+\left(\vec{u}_{s}\right)^{2}>0$

for all $s \in S_{+}$. In the following we use a diagonalization (3.17) obeying

$u_{0}^{s}>0, \quad u_{0}^{(i)}>0$

for all $s \in S$ and all $i$ such that $\xi_{i} \neq 0$ (and hence $d_{i} \neq 1$ ), where $u_{a}^{(i)}=S_{a}^{A} U_{A}^{(i)}$ are diagonalized curvature $U$-vectors, $i=1, \ldots, n$. The diagonalization (3.17) from [15] obeys these conditions and implies (3.28) and (3.29).

The inverse matrix $\left(S_{A}^{a}\right)=\left(S_{a}^{A}\right)^{-1}$ defines the map which is inverse to (3.17),

$z^{a}=S_{A}^{a} \sigma^{A}$,

$a=0, \ldots, N_{0}-1$. The inequalities (3.33) imply $\left|\vec{v}_{s}\right|>1$ for all $s \in S_{+}$. The potential $V_{\infty}$ corresponds to the billiard $B$ in the hyperbolic space $\left(D^{N_{0}-1}, h_{L}\right)$. This billiard is an open domain in $D^{N_{0}-1}$ obeying the set of inequalities

$\left|\vec{y}-\vec{v}_{s}\right|<\sqrt{\vec{v}_{s}^{2}-1}=r_{s}$,

$s \in S_{+}$. The boundary of the billiard $\partial B$ is formed by parts of hyper-spheres with centers in $\vec{v}_{s}$ and radii $r_{s}$.

The conditions (3.34) are obeyed for the diagonalization (3.35) with

$z^{0}=e_{A} \sigma^{A}$,

where $e=\left(e_{A}\right)$ is a normed timelike vector $(e, e)=-1$ obeying $\left(e, U^{\Lambda}\right)<0$ and $\left(e, U^{s}\right)<0$ for all $s \in S$. Hence $\left(e, U^{(i)}\right)<0$ for all $i$ obeying $\xi_{i} \neq 0$. Our choice in [15] was $e=U^{\Lambda} / \sqrt{\left|\left(U^{\Lambda}, U^{\Lambda}\right)\right|}$.

When all factor spaces $M_{i}$ are Ricci-flat, i.e. all $\xi_{i}=0$, the brane part of conditions (3.34) may be relaxed, while the curvature part of these conditions should be omitted. In this case we obtain a more general definition of the billiard walls (e.g. for $u_{s 0} \leq 0$ ) described in [39]. 
Thus, as in [36], we are led to the asymptotic relation for the function $\Psi_{0, L}\left(y^{0}, \vec{y}\right)$

$$
\left(-\frac{1}{2} \Delta[\bar{G}]+\delta V\right) \Psi_{0, L}=0
$$

with the zero boundary condition $\Psi_{0, L \mid \partial B}=0$ imposed.

Due to (3.20) we get $\Delta[\bar{G}]=-\left(\partial_{0}\right)^{2}+\Delta\left[h_{L}\right]$, where $\Delta\left[h_{L}\right]=\Delta_{L}$ is the Lapalace-Beltrami operator corresponding to the metric $h_{L}$.

By separating the variables,

$\Psi_{0, L}=\Psi_{0}\left(y^{0}\right) \Psi_{L}(\vec{y})$,

we obtain the following asymptotic equation (for $y^{0} \rightarrow$ $-\infty)$ :

$$
\left(\left(\frac{\partial}{\partial y^{0}}\right)^{2}+2 A e^{-2 y^{0}}+E-\frac{1}{4}\left(N_{0}-2\right)^{2}\right) \Psi_{0}=0
$$

where

$$
\Delta_{L} \Psi_{L}=-E \Psi_{L}, \quad \Psi_{L \mid \partial B}=0 .
$$

We assume that the minus Laplace-Beltrami operator $\left(-\Delta_{L}\right)$ with the zero boundary conditions has a spectrum obeying the following inequality:

$E \geq \frac{1}{4}\left(N_{0}-2\right)^{2}$.

This restriction was proved in $[27,28]$ for a wide class of billiards with finite volumes.

Here we restrict ourselves to the case of negative $A$ number $A<0$.

Solving Eq. (3.40) we get for $A<0$ the following set of basis solutions:

$\Psi_{0}=\mathcal{B}_{i \omega}\left(\sqrt{2|A|} e^{-y^{0}}\right)$,

where $\mathcal{B}_{i \omega}(z)=I_{i \omega}(z), K_{i \omega}(z)$ are the modified Bessel functions and

$\omega=\sqrt{E-\frac{1}{4}\left(N_{0}-2\right)^{2}} \geq 0$.

We denote

$U(\sigma)=U_{A} \sigma^{A}=\sum_{s \in S} U_{A}^{s} \sigma^{A}, \quad U_{A}=\sum_{s \in S} U_{A}^{s}$.

In the following we impose the restriction on $U=\left(U_{A}\right)$ : $(U, U)<0$. We have $\left(U, U^{\Lambda}\right)<0$ due to $\left(U^{s}, U^{\Lambda}\right)<0$ for all $s$.
From now we use a diagonalization with $z$-variables obeying (3.37) with

$e=U / \sqrt{|(U, U)|}$.

For $U=k U^{\Lambda}$, with $k>0$, such a diagonalization coincides with that of Ref. [15].

It was obtained in [36] that

$$
\Psi^{f_{0}} \sim C_{ \pm} \exp \left(\theta^{ \pm}(|\vec{y}|) e^{-y^{0}}-\frac{1}{2}(m-1) y^{0}\right) e^{i Q_{s} \Phi^{s}} \Psi_{L}(\vec{y}),
$$

as $y^{0} \rightarrow-\infty$ for any fixed $\vec{y} \in B$ and $C_{ \pm}$are non-zero constants, "plus" corresponds to $\mathcal{B}=I$ and "minus" to $\mathcal{B}=$ $K$. Here

$\theta^{ \pm}(|\vec{y}|)=-\frac{q}{2} \frac{\left(1+\vec{y}^{2}\right)}{\left(1-\vec{y}^{2}\right)} \pm \sqrt{-2 A}$

and

$q=\sqrt{-(U, U)}>0$.

Now we outline our analysis from [36] of asymptotic behavior of $\Psi^{f_{0}}$ as $y^{0} \rightarrow-\infty$. Here we fix all $\Phi^{s} \in \mathbb{R}$, $s \in S$.

For $\mathcal{B}=K, \Psi^{f_{0}} \rightarrow 0$ as $y^{0} \rightarrow-\infty$ for fixed $\vec{y} \in B$.

Now let $\mathcal{B}=I$.

If $\frac{1}{2} q>\sqrt{2|A|}$, or, equivalently,

$\sum_{s \in S}\left(U^{s}, U^{s}\right)<-(U, U)$,

we also get $\Psi^{f_{0}} \rightarrow 0$ as $y^{0} \rightarrow-\infty$ for fixed $\vec{y} \in B$.

For $\frac{1}{2} q=\sqrt{2|A|}$, or, equivalently,

$\sum_{s \in S}\left(U^{s}, U^{s}\right)=-(U, U)$

we get $\Psi^{f_{0}} \rightarrow 0$ as $y^{0} \rightarrow-\infty$ for fixed $\vec{y} \in B \backslash\{\overrightarrow{0}\}$. For $\vec{y}=\overrightarrow{0}$ we get $\left|\Psi^{f_{0}}\right| \rightarrow+\infty$ as $y^{0} \rightarrow-\infty$. It may be shown that in this case, when $m=N_{0}=n+l$ (i.e. if $m$ is the minimal number of walls which is necessary for the billiard to have a finite volume) we get

$\Psi^{f_{0}} \sim C_{0} \delta(\vec{y}) e^{i Q_{s} \Phi^{s}} \Psi_{L}(\overrightarrow{0})$,

as $y^{0} \rightarrow-\infty$, where $C_{0} \neq 0$ is a constant irrelevant for our consideration. Thus, for $m=n+l$ and for eigenfunction $\Psi_{L}(\vec{y})$ with $\Psi_{L}(\overrightarrow{0}) \neq 0$ we get a $\delta$-function in the asymptotic of $\Psi^{f_{0}}$. In this case we have an asymptotic localization of $\Psi^{f_{0}}$ at the point $\vec{y}=\overrightarrow{0}$ for our choice of gauge ("tortoise" one). 
When the scalar fields are absent and we use a diagonalization from $[33,40]$ the relation $\vec{y}=\overrightarrow{0}$ implies the isotropization $\beta^{i}=\beta$ and we may talk in terms of asymptotic quantum isotropization of the wave function in the temporal gauge under consideration.

When $\frac{1}{2} q<\sqrt{2|A|}$, or, equivalently,

$\sum_{s \in S}\left(U^{s}, U^{s}\right)>-(U, U)$

we get $\left|\Psi^{f_{0}}\right| \rightarrow+\infty$ as $y^{0} \rightarrow-\infty$ for $\vec{y}$ belonging to the open domain

$B_{\infty}=\left\{\vec{y} \in B:|\vec{y}|<\frac{2 \sqrt{2|A|}-q}{2 \sqrt{2|A|}+q}, \Psi_{L}(\vec{y}) \neq 0\right\}$.

Outside the closure of $B_{\infty}$ we get the zero limit of our wave function and we may talk in terms of the asymptotic localization of $\Psi^{f_{0}}$ in $B_{\infty}$.

With some exceptions we obtain the same results for the asymptotic behavior of the wave function in the harmonic gauge with $f=0: \Psi=e^{-b y^{0}} \Psi^{f_{0}}$ in the limit $y^{0} \rightarrow-\infty$, since the term $\left(-b y^{0}\right)$ in the exponent is suppressed generically by $e^{-y^{0}}$. The change of gauge (from tortoise to harmonic) may be sensitive for the asymptotic behavior of the $\Psi$-function in the case (3.51) if $\vec{y}=0$ and in the case (3.53) when $\vec{y}$ belongs to the border of the domain $B_{\infty}$.

\section{Example 1: $(n-1)$-dimensional billiards in the models with electric branes}

Here we illustrate our approach by considering the model with the Lagrangian

$\mathcal{L}=R[g]-2 \Lambda-\sum_{s=1}^{n} \frac{\theta_{s}}{n_{s} !}\left(F^{s}\right)_{g}^{2}+\Delta \mathcal{L}$.

Here we deal with the metric $g$ and the forms $F^{s}=d A^{s}$, $s=1, \ldots, n$, on the manifold $M$ from (2.3). We use the metric ansatz from (2.4) which deals with a warped product of the interval $\left(u_{-}, u_{+}\right)$and $n$ Einstein spaces. $\Delta \mathcal{L}$ is an extra term with fields of forms which will be specified below.

\subsection{The configuration with $n$ electric branes}

Here we put $\Delta \mathcal{L}=0$ and use the following non-composite electric ansatz for the fields of forms:

$F^{s}=\mathrm{d} \Phi^{s}(u) \wedge \tau_{s}$,

$s=1, \ldots, n$, where $n \geq 2$.
We put $\left(U^{s}, U^{s}\right)>0$ for all $s=1, \ldots, n$; by this we exclude the case $n=2$ with $\left(d_{1}, d_{2}\right)=(1, k),(k, 1)$. The restriction (3.3) reads

$\theta_{s} \varepsilon(s)>0$,

$s=1, \ldots, n$. According to these restrictions we get $\varepsilon(s)=$ +1 for an ordinary form field $F^{s}$ with $\theta_{s}>0$, which means that the factor space $\left(M_{s}, g^{s}\right)$ should be either Euclidean with the signature $(+, \ldots,+)$, or it should have an even number of timelike directions: $(-,-,+, \ldots,+)$ and so on. For a phantom form field $F^{s}$ with $\theta_{s}<0$ we should consider the metric $g^{s}$ with either pseudo-Euclidean signature $(-,+, \ldots,+)$, or with the signatures $(-, \ldots,-,+, \ldots,+)$, containing an odd number of minuses.

There are three cases here: a) $w=-1$ in (2.4) and all $\left(M_{s}, g^{s}\right)$ are Riemannian spaces $\left(\theta_{s}>0\right.$ for all $\left.s\right)$; b) $w=+1,\left(M_{1}, g^{1}\right)$ has the signature $(-,-,+, \ldots,+)$ and $\left(M_{s}, g^{s}\right)$ are $s>1$ are Riemannian $\left(\theta_{s}>0\right.$ for all $\left.s\right)$; c) $w=+1,\left(M_{1}, g^{1}\right)$ has the signature $(-,+, \ldots,+)$ and $\left(M_{s}, g^{s}\right)$ with $s>1$ are Riemannian $\left(\theta_{1}<0\right.$ and $\theta_{s}>0$ for $s>1$ ). The case a) describes cosmological solutions ( $S$ branes), while b) and c) may describe static solutions, e.g. with spherical, cylindrical, and other symmetries.

For our configuration of branes (when $d_{1}>1, d_{2}>1$ for $n=2)$ the billiard $B \subset H^{n}$ has a finite volume. Indeed, let us suppose that $B$ has an infinite volume. Then there exists a set of (real) Kasner-like parameters $\alpha=\left(\alpha_{1}, \ldots, \alpha_{n}\right)$ obeying the relations

$\sum_{i=1}^{n} d_{i} \alpha^{i}=\sum_{i=1}^{n} d_{i}\left(\alpha^{i}\right)^{2}=1$

and the inequalities [15]

$U^{s}(\alpha)=d_{s} \alpha^{s}>0$

$s=1, \ldots, n$. Equations (4.4) and (4.5) are not compatible: otherwise we get inequalities $0<\alpha_{s}<\left(\alpha_{s}\right)^{2}<1$, for all $s$, which contradict (4.4). This proves the finiteness of the billiard volume.

Let us consider the quasi-Cartan matrix [35]

$A_{s s^{\prime}}=2\left(U^{s}, U^{s^{\prime}}\right) /\left(U^{s^{\prime}}, U^{s^{\prime}}\right)$,

where the scalar products (2.26) read in our case

$\left(U^{s}, U^{s^{\prime}}\right)=d_{s} \delta_{s s^{\prime}}-\frac{d_{s} d_{s^{\prime}}}{D-2}$,

$s, s^{\prime}=1, \ldots, n$. Thus we are led to the matrix

$A_{s s}=2, \quad A_{s s^{\prime}}=-\frac{2 d_{s}}{D-2-d_{s^{\prime}}}, \quad s \neq s^{\prime}$,

$s, s^{\prime}=1, \ldots, n$. 
It will be proved in a separate publication that the matrix (4.8) is coinciding with the Cartan matrix of some hyperbolic Kac-Moody algebra in the following six cases (up to permutations of indices): i) $n=2,\left(d_{1}, d_{2}\right)=(2,2),(2,3),(3,3)$, ii) $n=3,\left(d_{1}, d_{2}, d_{3}\right)=(1,1,1),(1,1,2)$, iii) $n=4$, $\left(d_{1}, d_{2}, d_{3}, d_{4}\right)=(1,1,1,1)$.

According to the classification of hyperbolic KM algebras by Carbone et al. [26] only the ranks $n=2,3,4$ should be considered here, since for $n>4$ there are no Dynkin diagrams where all nodes are connected by lines.

For $n=2$ and $\left(d_{1}, d_{2}\right)=(2,2),(2,3),(3,3)$ we get in (4.8) the Cartan matrices of the rank-2 hyperbolic KM algebras $H_{2}\left(p_{1}, p_{2}\right)$ with $\left(p_{1}, p_{2}\right)=(4,4),(4,3),(3,3)$, respectively. Here we use the notation $H_{2}\left(p_{1}, p_{2}\right)$ for the hyperbolic KM algebra of rank 2 with the Cartan matrix defined by the relations $A_{12}=-p_{1}, A_{21}=-p_{2}$, where $p_{1}$ and $p_{2}$ are natural numbers obeying $p_{1} p_{2}>4$.

For $n=3$ and $\left(d_{1}, d_{2}, d_{3}\right)=(1,1,1)$ we obtain the hyperbolic KM algebra by the number 7 in the classification of Saçlioğlu [24] (see also [25]), which is number 80 in the table of Ref. [26]. In this case $A_{s s^{\prime}}=-2$ for all $s \neq s^{\prime}$. This KM algebra appears for Bianchi-IX cosmology and its billiard coincides with the Chitré one. In the quantum case this billiard was considered in numerous papers; see [7,8,27, 28,41] and references therein.

For our model with a diagonal metric we may mimic the never ending asymptotic behavior near the singularity for three scale factors of Bianchi-IX model when $w=-1$, $\varepsilon(1)=\varepsilon(2)=\varepsilon(3)=+1, \theta_{1}=\theta_{2}=\theta_{3}=+1$. In this case we deal with approaching a spacelike singularity in the $D=4$ model with three 2-forms. For $w=+1, \varepsilon(1)=-1$, $\varepsilon(2)=\varepsilon(3)=+1, \theta_{1}=-1, \theta_{2}=\theta_{3}=+1$ we find the never ending asymptotic behavior of the scale factors near a timelike singularity. In this case we have a phantom 2-form $F^{1}$ and two ordinary 2-forms $F^{2}, F^{3}$.

For the case $n=3, d_{1}=d_{2}=1, d_{3}=2$ we get the billiard corresponding to the hyperbolic KM algebra by number 40 in the classification of [26]. Here $A_{12}=A_{21}=-1, A_{13}=$ $A_{31}=A_{23}=A_{32}=-2$. We have a billiard of finite volume which may describe the never ending oscillating behavior near either spacelike or timelike singularity. For the case of a spacelike singularity we put $w=-1$ and use all metrics $g^{i}$ of Euclidean signatures and all forms are taken to be ordinary ones. For the case with a timelike singularity we have three (non-equivalent) possibilities with $w=1$ : (a) $g^{1}=-\mathrm{d} x^{1} \otimes$ $\mathrm{d} x^{1}, g^{2}=\mathrm{d} x^{2} \otimes \mathrm{d} x^{2}$, and $g^{3}$ has the signature $(+,+)(\mathrm{b})$ $g^{1}=\mathrm{d} x^{1} \otimes \mathrm{d} x^{1}, g^{2}=\mathrm{d} x^{2} \otimes \mathrm{d} x^{2}$, and $g^{3}$ is of signature $(-,+)$; (c) $g^{1}=\mathrm{d} x^{1} \otimes \mathrm{d} x^{1}, g^{2}=\mathrm{d} x^{2} \otimes \mathrm{d} x^{2}$, and $g^{3}$ is of signature $(-,-)$. In the first two cases only one form should be phantom: $F^{1}$ or $F^{3}$ in cases (a) or (b), respectively. In the case (c) all three forms are ordinary ones.

For the last example $n=4, d_{1}=d_{2}=d_{3}=d_{4}=1$ we get the hyperbolic KM algebra by number 124 from [26] with
$A_{s s^{\prime}}=-1$ for all $s \neq s^{\prime}$. For our model with four ordinary 2-forms we get a diagonal cosmological metric with $w=-1$ and $g^{i}=\mathrm{d} x^{i} \otimes \mathrm{d} x^{i}, i=1,2,3,4$, which describes a never ending oscillating behavior near the spacelike singularity. An analogous behavior will be obtained in approaching a timelike singularity, if $w=1, g^{1}=-\mathrm{d} x^{1} \otimes \mathrm{d} x^{1}, g^{i}=$ $\mathrm{d} x^{i} \otimes \mathrm{d} x^{i}, i=2,3,4$, when the only one 2-form, namely $F^{1}$, is phantom.

For the model under consideration the basis asymptotic solutions for the wave function are given by Eq. (3.22) with the prefactor

$C(\sigma)=\frac{1}{2}\left(\sum_{s=1}^{n} d_{s} \beta^{s}-n y^{0}\right)$,

and equations (3.22), (3.39), (3.41), (3.43), and (3.44) where the relation for $A$-number (3.26) reads

$$
\begin{aligned}
A= & \frac{1}{8(2 n-1)} \\
& \times\left[-\frac{D-1}{D-2}-(2 n-2) \sum_{s=1}^{n} d_{s}\left(1-\frac{d_{s}}{D-2}\right)\right]<0 .
\end{aligned}
$$

Since our diagonalization (4.13) uses a timelike co-vector $U^{\Lambda}$ which coincides with a sum of $n$ brane vectors $U=$ $U^{1}+\cdots+U^{n}$, the whole of our analysis of the asymptotic behavior from the previous section is relevant.

We get $\left|\Psi^{f_{0}}\right| \rightarrow 0$ as $y^{0} \rightarrow-\infty$ for the basis solutions with modified Bessel function $\mathcal{B}=K$. For the basis solutions with another choice of modified Bessel function $\mathcal{B}=I$ we obtain (generically) non-empty "spots" $B_{\infty} \subset B$ (see (3.54)) for some basis functions, where the $\left|\Psi^{f_{0}}\right| \rightarrow+\infty$. These "spots" appear in the model under consideration since the inequality

$\sum_{s=1}^{n}\left(U^{s}, U^{s}\right) \geq-(U, U)$

is valid for all sets $\left(d_{1}, d_{2}, \ldots, d_{n}\right)$ with the exception: $\left(d_{1}, d_{2}\right)=(1, k),(k, 1)$. Indeed, Eq. (4.11) in our case is equivalent to the relation

$$
\left(\sum_{s=1}^{n} d_{s}-2\right) \sum_{s=1}^{n} d_{s} \geq \sum_{s=1}^{n} d_{s}^{2}
$$

which could be readily proved for all sets with the exception $\left(d_{1}, d_{2}\right)=(1, k),(k, 1)$. The equality in (4.12) takes place only for $\left(d_{1}, d_{2}\right)=(2,2)$ and $\left(d_{1}, d_{2}, d_{3}\right)=(1,1,1)$. In this case we may have a point-like "spot" for $\vec{y}=\overrightarrow{0}$ and a delta-function localization of the wave function $\Psi^{f_{0}}$ for $y^{0} \rightarrow-\infty$. This singularity can be eliminated if we change 
to the harmonic gauge. In all other cases the radius of any "spot" is non-zero and the "spot" cannot be eliminated by a transition to the harmonic gauge. Equations (4.18), (4.21), when substituted into the general formulas of the previous section, will give a solution to the problem in the quantum case.

Here the asymptotic solution to WDW equation are found up to the spectrum of the (minus) Laplace-Beltrami operator (3.41) with the zero boundary conditions imposed. For the $n=2$ case this can be done explicitly.

The case $n=2$. Now we consider the case $n=2$, when $d_{1} \geq 2, d_{2} \geq 2$. We use the following diagonalization of variables:

$z^{0}=q^{-1}\left(d_{1} \beta^{1}+d_{2} \beta^{2}\right), \quad z^{1}=q_{1}^{-1}\left(\beta^{1}-\beta^{2}\right)$,

where $q=[(D-1) /(D-2)]^{1 / 2}$ and $q_{1}=[(D-$ $\left.1) /\left(d_{1} d_{2}\right)\right]^{1 / 2}$. The components of $U^{s}$-vectors in $z$-variables read

$u_{0}^{s}=\frac{d_{s}}{(D-2) q}, \quad u_{1}^{1}=q_{1}^{-1}, \quad u_{1}^{2}=-q_{1}^{-1}$.

For 1-dimensional vectors from (3.31) we get

$v^{1}=-R / d_{1}<-1, \quad v^{2}=R / d_{2}>1$.

For $\left(d_{1}, d_{2}\right)=(2,2)$ we get $\left(v^{1}, v^{2}\right)=(-\sqrt{3}, \sqrt{3})$. Thus we are led to the 1-dimensional billiard $B=\left(y_{1}, y_{2}\right)$ with point-like walls assigned to

$y_{1}=v^{1}+\sqrt{\left(v^{1}\right)^{2}-1}, \quad y_{2}=v^{2}-\sqrt{\left(v^{2}\right)^{2}-1}$,

which obey $-1<y_{1}<0$ and $0<y_{2}<1$. $B$ belongs to the $1 d$ unit "disk" $D^{1}=(-1,1)$, which is an image of the 1-dimensional hyperbolic space $H^{1} \subset \mathbb{R}^{1,1}$ under the stereographic projection from the point $\left(z^{0}, z^{1}\right)=(-1,0)$. The billiard is subcompact, i.e. its completion $\left[y_{1}, y_{2}\right]$ is compact. We get $\left(y_{1}, y_{2}\right)=(-\sqrt{3}+\sqrt{2}, \sqrt{3}-\sqrt{2})$ for $\left(d_{1}, d_{2}\right)=(2,2)$.

In the quantum case the model with two factor spaces is integrable in the asymptotic regime of the formation of billiard walls. Here we have a discrete spectrum of the Laplace-Beltrami operator on $B=\left(y_{1}, y_{2}\right)$ with the metric $h_{L}=4 \mathrm{~d} y \otimes \mathrm{d} y /\left(1-y^{2}\right)^{2}\left(y=y^{1}\right)$, when the zero boundary conditions at points $y_{1}, y_{2}$ are imposed. Making the coordinate transformation

$x(y)=\ln \frac{1+y}{1-y}$,

we reduce the metric to the simple form $h_{L}=\mathrm{d} x \otimes \mathrm{d} x$ and $\Delta_{L}=\mathrm{d}^{2} / \mathrm{d} x^{2}$. We get a discrete spectrum of the LaplaceBeltrami operator on $\left(x_{1}=x\left(y_{1}\right), x_{2}=x\left(y_{2}\right)\right)$ with the zero boundary conditions: $\Delta_{L} \Psi_{L, k}=-E_{k} \Psi_{L, k}, \Psi_{L, k}\left(x_{i}\right)=0$, $i=1,2$ :

$\Psi_{L, k}=C_{k} \sin \left(k \frac{x-x_{1}}{x_{2}-x_{1}} \pi\right), \quad E_{k}=k^{2} \pi^{2} /\left(x_{2}-x_{1}\right)^{2}$,

$k=1,2,3, \ldots$ Here the constants $C_{k} \neq 0$ are irrelevant for our consideration. The calculations give

$x_{2}-x_{1}=\ln \frac{\left(1+y^{2}\right)\left(1-y^{1}\right)}{\left(1-y^{2}\right)\left(1+y^{1}\right)}=\frac{1}{2} \ln Q$,

where

$Q=\frac{\left(v^{2}+1\right)\left(\left|v^{1}\right|+1\right)}{\left(v^{2}-1\right)\left(\left|v^{1}\right|-1\right)}=\frac{\left(R+d_{1}\right)\left(R+d_{2}\right)}{\left(R-d_{1}\right)\left(R-d_{2}\right)}$.

(For $\left(d_{1}, d_{2}\right)=(2,2)$ we get $x_{2}-x_{1}=\ln (2+\sqrt{3})$.) Hence the spectrum depends only on the parameter $Q$ and the quantum number $k$ :

$E_{k}=\frac{4 \pi^{2} k^{2}}{(\ln Q)^{2}}$

$k=1,2,3, \ldots$ In the symmetric case $d_{1}=d_{2}=d$ the eigenfunctions $\Psi_{L, k}$ have a zero at $x=0$, or, equivalently, at $y=0$, only for even $k$.

It may be shown that in the case $d_{1}=d_{2}=d \geq 4$ (i.e. for $D \geq 9$ ) the "spot" covers the billiard with the exception of zeros of $\Psi_{L, k}$. This means that $\left|\Psi^{f_{0}}(y)\right| \rightarrow+\infty$ as $y^{0} \rightarrow$ $-\infty$ for $\mathcal{B}=I$ and any $y$, obeying $\Psi_{L, k}(y) \neq 0$. We recall that $\left|\Psi^{f_{0}}(y)\right| \rightarrow 0$ as $y^{0} \rightarrow-\infty$ for $\mathcal{B}=K$ and any $y$. This is valid for $Q_{1} \neq 0, Q_{2} \neq 0$.

In the classical case the model with two factor spaces is also integrable in the asymptotic regime of the formation of billiard walls. This is considered in the appendix in detail.

\subsection{The configuration with $n+1$ electric branes}

We extend the model from the previous subsection by adding an extra term, which we put in the Lagrangian (4.1),

$\Delta \mathcal{L}=\frac{\theta_{0}}{n_{0} !}\left(F^{0}\right)_{g}^{2}$

where $F^{0}=\mathrm{d} A^{0}$ is an $D$-dimensional form, i.e. $n_{0}=D$. We supplement the electric ansatz (4.2) by the following relation:

$F^{0}=\mathrm{d} \Phi^{0}(u) \wedge \tau_{1} \wedge \cdots \wedge \tau_{n}$

We get an additional brane vector $U^{0}=U^{\Lambda}$ and hence $U=U^{0}+U^{1}+\cdots+U^{n}=2 U^{\Lambda}$. Since $\left(U^{0}, U^{0}\right)<$ 0 adding the term (4.22) to the Lagrangian (4.1) does not 
change the billiard and the asymptotic behavior of the scale factors (near the singularity).

Now the basis asymptotic solutions for the wave function from the previous subsection are modified by adding the new variable $\Phi^{0}$ and using another prefactor,

$C(\sigma)=\frac{1}{2}\left[2 \sum_{s=1}^{n} d_{s} \beta^{s}-(n+1) y^{0}\right]$,

and another $A$-number,

$A=\frac{1}{16 n}\left[(2 n-5) \frac{D-1}{D-2}-(2 n-1) \sum_{s=1}^{n} d_{s}\left(1-\frac{d_{s}}{D-2}\right)\right]$.

In this case the relations $d_{s} \leq D-3$ (following from $\left(U^{s}, U^{s}\right)>0$ ) imply $A \leq-\frac{1}{4 n} \frac{D-1}{D-2}<0$. Since the Anumber from (4.25) differs from (4.10) we are led to a different asymptotic behavior for the wave function $\Psi^{f_{0}} \rightarrow 0$ as $y^{0} \rightarrow-\infty$ in this case when the electric brane with the brane vector $U^{0}$ obeying $\left(U^{0}, U^{0}\right)<0$ is added.

Now we consider the relation for the "spots" (4.11). We get

$\sum_{s=1}^{n}\left(U^{s}, U^{s}\right)+\left(U^{\Lambda}, U^{\Lambda}\right) \geq-4\left(U^{\Lambda}, U^{\Lambda}\right)$,

or, equivalently,

$(D-7)(D-1) \geq \sum_{s=1}^{n} d_{s}^{2}$.

In this case we get a different restriction on the "spots" in comparison with Eq. (4.12). Indeed, due to (4.27) for $D \leq 7$ the "spots" are absent and hence $\left|\Psi^{f_{0}}\right| \rightarrow 0$ as $y^{0} \rightarrow-\infty$ for all basis solutions. Thus adding of the term (4.22) to the Lagrangian changes (drastically) the asymptotic behavior of the wave functions as $y^{0} \rightarrow-\infty$, while in the classical case this term is irrelevant for the asymptotic behavior.

\section{Example 2: 4-dimensional billiard in $D=11$ model with ten magnetic branes}

Now we consider the 11-dimensional model with the metric $g$ and ten 4-forms $F^{J}=\mathrm{d} A^{J}, J \in \Omega$. The Lagrangian reads

$\mathcal{L}=R[g]-2 \Lambda-\frac{1}{4 !} \sum_{J \in \Omega}\left(F^{J}\right)_{g}^{2}$.

Here the index set $\Omega$ consists of all subsets $J=\left\{j_{1}, j_{2}, j_{3}\right\} \subset$ $\{1,2,3,4,5\}$. The action (2.1) is defined on the $11 d$ manifold
$M=\left(u_{-}, u_{+}\right) \times M_{1} \times M_{2} \times M_{3} \times M_{4} \times M_{5}$,

with $d_{1}=d_{2}=d_{3}=d_{4}=d_{5}=2$. We consider the cosmological ansatz for metric (2.4) with $w=-1$ and five $2 d$ Einstein spaces $\left(M_{i}, g^{i}\right)$ of Euclidean signature $(+,+)$ :

$g=-e^{2 \gamma(t)} \mathrm{d} t \otimes \mathrm{d} t+\sum_{i=1}^{5} e^{2 \beta^{i}(t)} g^{i}$

and impose the magnetic ansatz for the fields of forms

$F^{J}=*\left(\mathrm{~d} \Phi^{J} \wedge \tau(J)\right)$,

$J \in \Omega$. Here $\Phi^{J}=\Phi^{J}(t), *$ is the Hodge operator and $\tau(J)=\tau_{j_{1}} \wedge \tau_{j_{2}} \wedge \tau_{j_{3}}, J=\left\{j_{1}, j_{2}, j_{3}\right\} \in \Omega, j_{1}<j_{2}<$ $j_{3}$. For $\Lambda=0$ we deal with 10 non-composite magnetic branes which mimic $S M 5$-branes in "truncated" $D=11$ supergravity without Chern-Simons term.

It was proved in [15] that the billiard $B \subset H^{4}$ has a finite volume.

For all $J$ we get $d(J)=6,\left(U^{J}, U^{J}\right)=2$ and $U=$ $\sum_{J} U^{J}=6 U^{\Lambda}$ and hence $(U, U)=-40$. Here $N=15$. We obtain

$C(\sigma)=6 \sum_{s=1}^{5} \beta^{s}-5 y^{0}$

for the prefactor and

$A=-\frac{75}{28}<0$

for the $A$-number.

In this case we get $\sum_{s}\left(U^{J}, U^{J}\right)<-(U, U)$ and hence $\left|\Psi^{f_{0}}\right| \rightarrow 0$ as $y^{0} \rightarrow-\infty$ for all basis solutions. In the harmonic time gauge we also get $\left|\Psi^{f=0}\right| \rightarrow 0$ as $y^{0} \rightarrow-\infty$ for all basis solutions.

Thus in this example we have a similar asymptotic behavior of the basis wave functions to the case of $9 d$ billiards with a maximal number of $S M$-branes, either electric [29] or electric plus magnetic [36].

\section{Conclusions}

We have generalized the quantum billiard approach from [36] by considering a cosmological-type model with $n$ Einstein factor spaces in the theory with several forms, $l$ scalar fields and a $\Lambda$-term. As in [36], after imposing the electromagnetic composite brane ansatz with certain restrictions for brane intersections and parameters of the model we have used the Wheeler-DeWitt (WDW) equation for the model, written in the conformally covariant form. 
By imposing restrictions on the parameters of the model, e.g. on brane $U^{s}$-vectors and using the vanishing of the potential terms coming from curvatures of the Einstein spaces and the $\Lambda$-term [15], we have obtained the asymptotic solutions to the WDW equation, in the limit of formation of billiard walls: $y^{0} \rightarrow-\infty$, which have a form similar to that from [36].

We have studied a subclass of examples of classical and quantum billiards in the model with $n$ non-intersecting electric branes, e.g., for certain hyperbolic KM algebras of ranks $n=2,3,4$. In the classical case any of these billiards $B$ has a finite volume and describes a never ending oscillatory behavior of the scale factors while approaching a singularity, which may be either spacelike or timelike.

In the quantum case the asymptotic basis solutions to the WDW equation in the "tortoise" time gauge tends to zero: $\Psi^{f_{0}} \rightarrow 0$, as $y^{0} \rightarrow-\infty$, for the following choice of the Bessel function: $\mathcal{B}=K$, while for another choice of the Bessel function, $\mathcal{B}=I$, we have obtained for some basis solutions $\left|\Psi^{f_{0}}\right| \rightarrow+\infty$ when $y^{0} \rightarrow-\infty$ in certain domain $B_{\infty} \subset B$ of non-zero measure - a "spot" - for all cases but $\left(d_{1}, d_{2}\right)=(2,2)$ and $\left(d_{1}, d_{2}, d_{3}\right)=(1,1,1)$. The "spot" does not disappear in the generic cases when the wave function in the harmonic time gauge is considered. For two exceptional cases we have a point-like "spot" at $\vec{y}=\overrightarrow{0}$ for some basis solutions in the "tortoise" time gauge, which corresponds to $\delta$-functions in the limit $y^{0} \rightarrow-\infty$, but this singularity does not take place in the harmonic time gauge. For $n=2$ we have found the asymptotic solutions for the metric in the classical case (see the appendix) as well as its quantum counterpart, i.e. the asymptotic (basis) solutions to the WDW equation.

Here we have considered the branes with general scalar products $\left(U^{s}, U^{s}\right)$, while in [36] the restriction $\left(U^{s}, U^{s}\right)>0$ was used. The presence of branes with $\left(U^{s}, U^{s}\right) \leq 0$ has no effect on the billiard $B$ and the asymptotic classical behavior of scale factors and scalar fields (as $y^{0} \rightarrow-\infty$ ) but it changes the asymptotic solutions to WDW equation. We have illustrated this effect by an example with $n$ electric branes on product of $n$ Einstein spaces obeying $\left(U^{s}, U^{s}\right)>0$ and one brane obeying $\left(U^{0}, U^{0}\right)<0$. It is shown that for such configuration of branes the "spots" disappear for $D \leq 7$ and hence $\left|\Psi^{f_{0}}\right| \rightarrow 0$ as $y^{0} \rightarrow-\infty$ for all basis solutions to the WDW equation. The analogous asymptotic behavior of all basis solutions $\Psi^{f_{0}}$ is shown to be valid for the 4dimensional quantum billiard in the $D=11$ model with ten magnetic branes, which was considered earlier for the classical case in [15]. This result can be extended to the configuration with composite magnetic S-branes in the model with one 4-form, but an open problem here is to include the ChernSimons term of $D=11$ supergravity into the consideration. In the classical case there were some obstacles for doing so [15].
Recently, a certain interest in studying a possible oscillating behavior near a timelike singularity, started by Parnovsky [5,6], appeared after Refs. [42,43]. In [43] the authors speculated that such singularities, if occurring in AdS/CFT and being of the chaotic variety, may be interpreted as transient chaotic renormalization group flows which exhibit features reminiscent of chaotic duality cascades. So, the examples of billiards describing an oscillating behavior near a timelike singularity, which were considered in this paper, may be tested for a possible application to the program suggested in Ref. [43].

Acknowledgments This paper was financially supported by the Ministry of Education and Science of the Russian Federation on the program to improve the competitiveness of Peoples Friendship University among the world's leading research and education centers in the years 20162020. The author thanks T. Damour for hospitality in IHES (Bures-surYvette), where this work was started, and also thanks H. Nicolai for hospitality in AEI (Golm), where this work was finished. The author thanks V.A. Belinskii and A. Kleinschmidt for useful discussions.

Open Access This article is distributed under the terms of the Creative Commons Attribution 4.0 International License (http://creativecomm ons.org/licenses/by/4.0/), which permits unrestricted use, distribution, and reproduction in any medium, provided you give appropriate credit to the original author(s) and the source, provide a link to the Creative Commons license, and indicate if changes were made. Funded by SCOAP ${ }^{3}$.

\section{Appendix 1. Classical asymtotical solution for $\boldsymbol{n}=2$}

In the classical case the motion of a point-like particle in the billiard from Sect. 4 describes an asymptotic solution for the metric with "jumping” Kasner-like parameters,

$g_{\text {as }}=w \mathrm{~d} \tau \otimes \mathrm{d} \tau+\sum_{i=1}^{n} B_{i}^{2}(\tau) \tau^{2 \alpha^{i}(\tau)} g^{i}, \quad w= \pm 1$

It is smooth when the synchronous-like variable $\tau$ belongs to intervals $\left(\tau_{1}, \tau_{2}\right),\left(\tau_{2}, \tau_{3}\right), \ldots$, where $\tau_{1}>\tau_{2}>\tau_{3}>\cdots>$ 0 is an (in general unknown) sequence of points tending to 0 , and the sets of real functions $B(\tau)=\left(B_{i}(\tau)>0\right), \alpha(\tau)=$ $\left(\alpha^{i}(\tau)\right)$ take constant values on these intervals, i.e. $B(\tau)=$ $\left(B_{i}^{(k)}\right), \alpha(\tau)=\left(\alpha_{(k)}^{i}\right)$ for $\tau \in\left(\tau_{k}, \tau_{k+1}\right), k=1,2, \ldots$, while the scale factors $a_{i}(\tau)=B_{i}(\tau) \tau^{\alpha^{i}(\tau)}, i=1,2$, are continuous functions on $\left(\tau_{1}, 0\right)$. All values $\left(\alpha_{(k)}^{i}\right)$ obey the Kasner-like equations (4.4). The points $\tau_{1}, \tau_{2}, \tau_{3}, \cdots>0$ correspond to collisions with walls corresponding to branes. An impact with an $s$-wall in the billiard leads to a change of the Kasner-like set $\alpha \mapsto \hat{\alpha}$ [44].

Here the following inequalities should be valid: $U^{s}(\alpha)=$ $d_{s} \alpha^{s}<0$ (before a collision with an $s$-wall), $U^{s}(\hat{\alpha})=$ $d_{s} \hat{\alpha}^{s}>0$ (after a collision with an $s$-wall). 
The case $n=2$. Let us consider the case $n=2$, when $d_{1} \geq 2, d_{2} \geq 2$. We obtain only two sets of solutions to Eqs. (4.4) [40],

$\alpha_{ \pm}^{1}=\frac{d_{1} \pm \sqrt{R}}{d_{1}\left(d_{1}+d_{2}\right)}, \quad \alpha_{ \pm}^{2}=\frac{d_{2} \mp \sqrt{R}}{d_{2}\left(d_{1}+d_{2}\right)}$,

where

$$
R=\sqrt{d_{1} d_{2}\left(d_{1}+d_{2}-1\right)} .
$$

Here the plus sign corresponds to the motion from the first wall with $s=1$ to the second one with $s=2$, and the minus sign vice versa.

Let us consider the billiard chamber $B_{c h}$, which is an open domain in the lower light cone defined by the relations

$u_{0}^{s} z^{0}+u_{1}^{s} z^{1}<0, \quad s=1,2, \quad z^{0}<-\left|z^{1}\right|$.

By the transformations (3.18), (3.19) $B_{c h}$ is projected onto $B$. Any border line $L_{s}$ obeying $u_{0}^{s} z^{0}+u_{1}^{s} z^{1}=0$, or, equivalently,

$z^{1}=c_{s} z^{0}, \quad c_{s}=\left(v^{s}\right)^{-1}$,

is projected onto a point $y_{s}, s=1,2$. The lines $L_{1}$ and $L_{2}$ may be considered as world lines of point-like mirrors moving with the velocities $-1<c_{1}<0$ and $0<c_{2}<1$. (Here, for the speed of light we put $c=1$.) The asymptotic motion in the billiard $B$ with a sequence of bounce points: $y_{1}, y_{2}, y_{1}, \ldots$ is a projection of a zigzag world line of a light beam in the billiard chamber $B_{c h}$ with bounce points: $z_{1} \in L_{1}, z_{2} \in L_{2}, z_{3} \in L_{1}, \ldots$ For the first part of our world line we get $z_{2}^{0}-z_{1}^{0}=z_{2}^{1}-z_{1}^{1}$ with $z_{1}^{1}=c_{1} z_{1}^{0}$ and $z_{2}^{1}=c_{2} z_{2}^{0}$, which implies

$z_{2}^{0}=Q_{+} z_{1}^{0}, \quad Q_{+}=\frac{1-c_{1}}{1-c_{2}}>1$

For the second part of the world line we obtain $z_{3}^{0}-z_{2}^{0}=$ $-\left(z_{3}^{1}-z_{2}^{1}\right)$ with $z_{3}^{1}=c_{1} z_{3}^{0}$ (and $\left.z_{2}^{1}=c_{2} z_{2}^{0}\right)$, which gives us

$z_{3}^{0}=Q_{-} z_{2}^{0}=Q_{-} Q_{+} z_{1}^{0}, \quad Q_{-}=\frac{1+c_{2}}{1+c_{1}}>1$

Thus for the $z^{0}$-components of the bounce points we have

$z_{1}^{0}, \quad z_{2}^{0}=Q_{+} z_{1}^{0}, \quad z_{3}^{0}=Q_{-} Q_{+} z_{1}^{0}$,

$z_{4}^{0}=Q_{+} Q_{-} Q_{+} z_{1}^{0}, \ldots$,

which may be written as follows:

$z_{2 k+1}^{0}=Q^{k} z_{1}^{0}, \quad z_{2 k}^{0}=Q_{+} Q^{k-1} z_{1}^{0}, \quad Q=Q_{-} Q_{+}>1$, for $k=1,2, \ldots$.

In terms of dimensions $d_{i}$ the parameters $Q_{-}, Q_{+}$read

$Q_{+}=\frac{R+d_{1}}{R-d_{2}}, \quad Q_{-}=\frac{R+d_{2}}{R-d_{1}}$

where $R=R\left(d_{1}, d_{2}\right)$ is defined in (A.3).

Now we use the asymptotic relation for the volume scale factor $v=\exp \left(d_{1} \beta^{1}+d_{2} \beta^{2}\right)$ following from (A.1) and (4.13)

$v=\exp \left(q z_{0}\right)=C \tau$

where $C$ is the integration constant, $C=B_{1}^{d_{1}} B_{2}^{d_{2}}$ for all $\tau$. We put $\tau_{1}$ to be sufficiently small, i.e. obeying at least the restriction $C \tau_{1}<1$. Then using (A.9) and (A.11) we get the asymptotic relation for a set of bounce points $\tau_{1}>\tau_{2}>$ $\tau_{3}>\cdots>0$, when $\tau_{1}$ corresponds to a collision with the first wall:

$\ln \left(C \tau_{2 k+1}\right)=Q^{k} \ln \left(C \tau_{1}\right), \quad \ln \left(C \tau_{2 k}\right)=Q_{+} Q^{k-1} \ln \left(C \tau_{1}\right)$,

for $k=1,2, \ldots$ and $C \tau_{1}<1$.

Using the continuity conditions for the scale factors at the points $\tau_{k}$ we get for $B\left(\tau_{k}-0\right)=B_{i}^{(k)}, i=1,2$ :

$B_{i}^{(2)}=\tau_{2}^{\Delta^{i}} B_{i}^{(1)}, \quad B_{i}^{(3)}=\tau_{3}^{-\Delta^{i}} B_{i}^{(2)}=\tau_{2}^{\Delta^{i}} \tau_{3}^{-\Delta^{i}} B_{i}^{(1)}, \ldots$

where $\Delta^{i}=\alpha_{+}^{i}-\alpha_{-}^{i}$, or

$\Delta^{1}=\frac{\Delta_{0}}{d_{1}}, \quad \Delta^{2}=-\frac{\Delta_{0}}{d_{2}}, \quad \Delta_{0}=\frac{2 \sqrt{R}}{d_{1}+d_{2}}$.

We get from (A.13)

$\ln \left(B_{i}^{(2 k+1)} / B_{i}^{(1)}\right)=\Delta^{i} Q_{+}\left(1-Q_{-}\right) \frac{Q^{k}-1}{Q-1} \ln \left(C \tau_{1}\right)$,

$\ln \left(B_{i}^{(2 k)} / B_{i}^{(1)}\right)=-\Delta^{i} \ln C+\Delta^{i}\left[\left(Q_{+}-1\right) \frac{Q^{k}-1}{Q-1}+1\right]$

$\ln \left(C \tau_{1}\right)$,

for $i=1,2$ and $k=1,2, \ldots$ Thus, we obtain an asymptotic (oscillating) solution for the metric with two scale factors. It does not depend on $\Lambda$, the curvatures of the Einstein spaces and the brane charge densities. This approximation works for small enough value of the parameter $C \tau_{1}=\delta$. 


\section{References}

1. D.M. Chitré, Investigation of vanishing of a horizon for Bianchi type IX (Mixmaster) Universe. Ph.D. thesis, University of Maryland, Technical Report No. 72-125 (1972)

2. V.A. Belinskii, E.M. Lifshitz, I.M. Khalatnikov, Usp. Fiz. Nauk 102, 463 (1970). (in Russian)

3. V.A. Belinskii, E.M. Lifshitz, I.M. Khalatnikov, Adv. Phys. 31, 639 (1982)

4. C.W. Misner, Quantum cosmology. Phys. Rev. 186, 1319 (1969)

5. S.L. Parnovsky, Gravitation fields near the naked singularities of the general type. Phys. A Stat. Mech. Appl. 104(1-2), 210-222 (1980)

6. S.L. Parnovsky, A general solution of gravitational equations near their singularities. Class. Quant. Grav. 7(4), 571-575 (1990)

7. R. Graham, P. Szepfalusy, Quantum creation of the generic universe. Phys. Rev. D 42, 2483-2490 (1990)

8. A.A. Kirillov, On quantum properties of the large scale inhomogeneities of metric in the vicinity of cosmological singularity. Int. J. Mod. Phys. D 3, 431-441 (1994)

9. B.S. DeWitt, Quantum theory of gravity. I. The canonical theory. Phys. Rev. 160, 1113-1148 (1967)

10. V.D. Ivashchuk, A.A. Kirillov, V.N. Melnikov, On stochastic properties of multidimensional cosmological models near the singular point. Izv. Vuzov (Fizika) 11, 107 (1994). (in Russian)

11. V.D. Ivashchuk, A.A. Kirillov, V.N. Melnikov, Russ. Phys. J. 37, 1102 (1994)

12. V.D. Ivashchuk, V.N. Melnikov, Billiard representation for multidimensional cosmology with multicomponent perfect fluid near the singularity. Class. Quant. Grav. 12(3), 809-826 (1995). arXiv:gr-qc/9407028

13. A.A. Kirillov, Reduction of additional dimensions in nonuniform quantum Kaluza-Klein cosmological model. JETP Lett. 62, 89 (1995)

14. A.A. Kirillov, Pisma. Zh. Eksp. Teor. Fiz. 62, 81 (1995)

15. V.D. Ivashchuk, V.N. Melnikov, Billiard representation for multidimensional cosmology with intersecting p-branes near the singularity. J. Math. Phys. 41(9), 6341-6363 (2000). arXiv:hep-th/9904077

16. V.D. Ivashchuk, V.N. Melnikov, D. Singleton, On avoiding cosmological oscillating behavior for S-brane solutions with diagonal metrics. Phys. Rev. D 72, 103511 (2005). arXiv:gr-qc/0509065

17. T. Damour, M. Henneaux, Chaos in superstring cosmology. Phys. Rev. Lett. 85, 920-923 (2000). arXiv:hep-th/0003139

18. T. Damour, M. Henneaux, Oscillatory behaviour in homogeneous string cosmology models. Phys. Lett. B 488, 108-116 (2000)

19. T. Damour, M. Henneaux, Erratum to: Oscillatory behaviour in homogeneous string cosmology models. Phys. Lett. B 491, 377 (2000)

20. T. Damour, M. Henneaux, H. Nicolai, Cosmological billiards, topical review. Class. Quantum Grav. 20, R145-R200 (2003). arXiv:hep-th/0212256

21. T. Damour, M. Henneaux, $E_{10}, B E_{10}$ and arithmetical chaos in superstring cosmology. Phys. Rev. Lett. 86, 4749-4752 (2001). arXiv:hep-th/0012172

22. M. Henneaux, D. Persson, P. Spindel, Spacelike singularities and hidden symmetries of gravity. Liv. Rev. Relativ. 11, 1-228 (2008)

23. V.G. Kac, Infinite-Dimensional Lie Algebras (Cambridge University Press, Cambridge, 1990)

24. C. Saçlioğlu, Dynkin diagram for hyperbolic Kac-Moody algebras. J. Phys. A 22(18), 3753-3769 (1989)
25. S. de Buyl, C. Schomblond, Hyperbolic Kac-Moody algebras and Einstein billiards. J. Math. Phys. 45, 4464-4492 (2004). arXiv:hep-th/0403285

26. L. Carbone, S. Chung, L. Cobbs, R. McRae, D. Nandi, Y. Naqvi, D. Penta, Classification of hyperbolic Dynkin diagrams, root lengths and Weyl group orbits. J. Phys. A Math. Theor. 43, 155209 (2010). arXiv: 1003.0564

27. A. Kleinschmidt, M. Koehn, H. Nicolai, Supersymmetric quantum cosmological billiards. Phys. Rev. D 80, 061701 (2009). arXiv:0907.3048

28. A. Kleinschmidt, H. Nicolai, Cosmological quantum billiards. arXiv:0912.0854

29. V.D. Ivashchuk, V.N. Melnikov, Quantum billiards in multidimensional models with fields of forms. Grav. Cosmol. 19(3), 171-177 (2013). arXiv:1306.6521

30. H. Nicolai, Quantum gravity: the view from particle physics. arXiv: 1301.5481

31. C.W. Misner, Minisuperspace. In: Klauder, J.R. (ed.) Magic Without Magic, pp. 441-473 (John Archibald Wheeler, Freeman, San Francisko, 1972)

32. J.J. Halliwell, Derivation of the Wheeler-De Witt equation from a path integral for minisuperspace models. Phys. Rev. D 38, 2468 (1988)

33. V.D. Ivashchuk, V.N. Melnikov, A.I. Zhuk, On Wheeler-DeWitt equation in multidimensional cosmology. Nuovo Cimento B 104(5), 575-581 (1989)

34. P. Hájiček, K.V. Kuchař, Constraint quantization of parametrized relativistic gauge systems in curved spacetimes. Phys. Rev. D 41, 1091-1104 (1990)

35. V.D. Ivashchuk, V.N. Melnikov, Multidimensional classical and quantum cosmology with intersecting $p$-branes. J. Math. Phys. 39 2866-2889 (1998). arXiv:hep-th/9708157

36. V.D. Ivashchuk, V.N. Melnikov, Quantum billiards in multidimensional models with branes. Eur. Phys. J. C 74, 2805 (2014). arXiv: 1310.4451

37. V.D. Ivashchuk, V.N. Melnikov, Sigma-model for the generalized composite p-branes. Class. Quantum Grav. 14, 30013029 (1997). (Corrigendum ibid. 15, 3941-3942 (1998)). arXiv:hep-th/9705036

38. V.D. Ivashchuk, V.N. Melnikov, Quantum billiards in multidimensional models with fields of forms on product of Einstein spaces. Grav. Cosmol. 22(2), 166-178 (2016)

39. V.D. Ivashchuk, V.N. Melnikov, On billiard approach in multidimensional cosmological models. Grav. Cosmol. 15(1), 49-58 (2009). arXiv:0811.2786

40. U. Bleyer, V.D. Ivashchuk, V.N. Melnikov, A.I. Zhuk, Multidimensional classical and quantum wormholes in models with cosmological constant. Nucl. Phys. B 429, 177-204 (1994)

41. O.M. Lecian, Periodic orbits in cosmological billiards: the Selberg trace formula for asymptotic Bianchi IX universes, evidence for scars in the wavefunction of the quantum universe and large-scale structure anisotropies of the present universe. arXiv:1311.0488

42. P. Klinger, Timelike singularities and Hamiltonian cosmological billiards. arXiv: 1512.03302

43. E. Shaghoulian, H. Wang, Timelike BKL singularities and chaos in $A d S / C F T$. arXiv: 1601.02599

44. V.D. Ivashchuk, On exact solutions in multidimensional gravity with antisymmetric forms. In: V. de Sabbata, G.T. Gillies, V.N. Melnikov (eds.) The Gravitational Constant: Generalized Gravitational Theories and Experiments, pp. 205-231. (Kluwer Academic Publ., Dordrecht, 2004). arXiv:gr-qc/0310114 